\title{
EDITORIAL
}

\section{Vaccine delivery systems}

\author{
Ryan F. Donnelly \\ School of Pharmacy, Queen's University Belfast, Belfast, UK
}

ARTICLE HISTORY Received 4 November 2016; Revised 4 November 2016; Accepted 5 November 2016

Vaccination is the most effective means of controlling infectious disease-related morbidity and mortality. The World Health Organization (WHO) estimates that vaccination prevents over 2.5 million child deaths each year worldwide. A vaccine is a biological preparation that improves immunity to a particular disease. The traditional types of vaccines that have been used to date clinically are vaccines that contain either dead or live-attenuated microorganisms, inactivated toxins (Toxoid), protein subunits, and polysaccharide antigens or conjugates. A number of innovative vaccines are in development such as recombinant vector and DNA vaccines. These agents resemble a disease-causing microorganism and stimulate the body's immune system to recognize the agent as foreign, destroy it, and "remember" it, so that the immune system can more easily challenge these microorganisms upon subsequent encounters.

Appropriate vaccine administration/delivery is the key element to ensure successful vaccination. Typically, most vaccines are administered via the subcutaneous (SC) or intramuscular (IM) routes. Hypodermic injections are associated with pain and distress that might lead to poor patient compliance and require highly trained personnel for administration. They are associated with a risk of disease transmission due to the possibility of needle-stick injuries or reuse of contaminated needles. Insufficient vaccine supply or limitation of vaccine production may also prove problematic in instances when mass vaccination is necessary. ${ }^{1,2}$

At present, most vaccines are deposited into the subcutaneous fat or into the muscle beneath the skin. Relatively few vaccines are administered into the viable skin (epidermis and dermis). ${ }^{3-5}$ Each of these routes of application relies on the presence of dendritic cells (DCs) in the tissues that take up the antigen, process it and present it to $\mathrm{T}$ lymphocytes in the draining lymphoid organs. Whereas subcutaneous fat and muscle tissue contain relatively few DCs, the dermis and the epidermis are densely populated by different subsets of DCs. Consequently, antigen delivery by hypodermic injection will bypass the skin's immune cells leading to less efficient vaccination. For this reason, the skin represents an ideal site for vaccine delivery, as vaccination at this site will evoke strong immune responses at much lower doses of antigen than intramuscular vaccines. ${ }^{6}$ Other areas of the body have also recently shown promise as targets for vaccine delivery, including the nasal mucosa and the gastrointestinal tract. These alternate sites of delivery offer the prospect for eliciting immune responses that are qualitatively different from those of injected vaccines or that stimulate immune responses at these mucosal sites for more effective defense against pathogens that invade by these routes, e.g., oral or nasal.

Vaccines formulated as liquids for injection are not ideal for the developing world in terms of need for a 'cold-chain'. Concerted efforts by researchers on alternative vaccine delivery routes have yielded a range of novel delivery devices with potential to enhance immunogenicity and stability.

In this Special Focus, experts in the field describe recent innovations in the design, evaluation and use of novel vaccine delivery devices and systems. In particular, they describe novel polymeric microneedle systems for delivery of DNA vaccines and review nasal and oral delivery systems for vaccines, paying particular attention to delivery system design, packaging and ease of administration. The development of vaccine adjuvants and the emerging area of nanoparticle-based cancer immunotherapeutics are also discussed in detail.

Nathalie Garçon and Alberta Di Pasquale (p 19) review the history of adjuvant use in vaccination and describe how new adjuvants and new combinations of adjuvants have opened the door to the delivery of improved and new vaccines against reemerging and difficult pathogens. Helmy Yusuf and Vicky Kett (p 34) discuss the concept of nasal vaccination, covering delivery system design and detailing the challenges and opportunities for the future, include translating in vivo data to clinical outcomes. Manjari Lal and Courtney Jarrahian (p 46) describe how oral administration of vaccines is simpler and more acceptable than injection via needle and syringe, particularly for infants, but caution that vaccine antigens and adjuvants given orally need buffering against the degradative effects of low stomach $\mathrm{pH}$ and the type and volume of antacid buffer require special attention for infants. In addition, they advise that container/closure systems must be compatible with vaccine formulations, protect against water and gas transfer and have minimal impact on the cold chain. Helen McCarthy and colleagues ( $p 50$ ) examine the ability of 4 polymers to formulate mechanically robust, functional DNA-loaded dissolvable microneedles. They show that complexation of DNA to a cationic delivery peptide prior to incorporation into the dissolvable microneedle matrix improves transfection efficiency following subsequent release. Yimei Jia and co-workers (p 63) review the use of multifunctional nanoparticles, such as 
liposomes, polymers, micelles, dendrimers, inorganic nanoparticles and hybrid nanoparticles, which have the potential to combine the delivery of a diverse range of therapeutic immunomodulators to increase the efficacy of tumor cell killing.

I have enjoyed reviewing these excellent papers, and I hope that readers find them both interesting and useful.

\section{Disclosure of potential conflicts of interest}

No potential conflicts of interest were disclosed.

\section{References}

[1] Hegde NR, Kaveri SV, Bayry J. Recent advances in the administration of vaccines for infectious diseases: Microneedles as painless delivery devices for mass vaccination. Drug Discov Today 2011; 16(23-24):1061-8; PMID:21782969; http://dx.doi.org/10.1016/j. drudis.2011.07.004
[2] Koutsonanos DG, del PilarMartin M, Zarnitsyn VG, Sullivan SP, Compans RW, Prausnitz MR, Skountzou I. Transdermal influenza immunization with vaccine-coated microneedle arrays. PLoS One 2009; 4(3):e4773; PMID:19274084; http://dx.doi.org/10.1371/journal. pone. 0004773

[3] Nicolas JF, Guy B. Intradermal, epidermal and transcutaneous vaccination: From immunology to clinical practice. Expert Rev Vaccines 2008; 7(8):1201-14; PMID:18844594; http://dx.doi.org/10.1586/ 14760584.7.8.1201

[4] Warger T, Schild H, Rechtsteiner G. Initiation of adaptive immune responses by transcutaneous immunization. Immunol Lett 2007; 109(1):13-20; PMID:17320194; http://dx.doi.org/ 10.1016/j.imlet.2007.01.007

[5] Stoitzner P, Sparber F, Tripp CH. Langerhans cells as targets for immunotherapy against skin cancer. Immunol Cell Biol 2010; 88 (4):431-7; http://dx.doi.org/10.1038/icb.2010.31

[6] Kenney RT, Yu J, Guebre-Xabier M, Frech SA, Lambert A, Heller BA, Ellingsworth LR, Eyles JE, Williamson ED, Glenn GM. Induction of protective immunity against lethal anthrax challenge with a patch. J Infect Dis 2004; 190(4):774-82; PMID:15272406; http://dx.doi.org/ $10.1086 / 422694$ 